\title{
Effects of Cubic Container's Wall or Floor on Random Packing Structures of Spherical Particles*
}

\author{
Isao TAGUCHI ${ }^{* *}$, Michio KURASHIGE ${ }^{* * *}$ and Kazuwo IMAI ${ }^{* * *}$
}

\begin{abstract}
By computer simulation, we examined how a wall or a floor of a container affects the random packing structure of a cubic aggregate of spherical particles. We constructed a model of the aggregate by sequential accumulation of spheres in a cubic box using (A) cyclic boundary conditions only in the $y$-direction together with random ups and downs of the box floor to estimate the wall effect and (B) the conditions in both of the horizontal directions to extract the floor effect. Using the obtained packing data, we estimated the "area porosities" and cumulative frequencies of the diameters of circles appearing on some cross sections created by cutting the aggregate at different levels, both as functions of distance from the wall/floor. From these results, we found that the surface effect seems to extend as deep as 2.5 particle diameters from the wall and approximately 4.5 diameters from the floor.
\end{abstract}

Key Words: Simulation, Sintered Material, Wall/Floor Effect, Random Packing, Packing Structure, Area Porosity

\section{Introduction}

Porous materials such as porous ceramics are important in various applications in industry. Particle-aggregate sintering is one of the most common and easiest methods of manufacturing such porous materials. Any manufacturing of particle aggregates and any experiment on them cannot avoid some structural influence of the wall and floor of the container into which the particles are poured. This layer affected by the wall or floor must be removed from the sintered particle aggregate in some applications and experiments. Therefore, we need to know the depth of the layer and how wall/floor affects packing structure.

Roblee et al. ${ }^{(1)}$ studied the influence of the confining wall of a cylindrical container on porosity in packed beds. Macrae and Gray ${ }^{(2)}$ showed that the effect of a wall on the packing structure in its vicinity is critically dependent on the conditions of deposition. Benenati and Brosilow ${ }^{(3)}$ presented a similar wall effect for a number of cases with the ratio varying from 2.6 to infinity of the diameter of a cylindrical container to that of the spheres poured into it and concluded that the extent of the wall effect ranges

\footnotetext{
* Received 2nd May, 2005 (No. 05-4034)

** Graduate School of Engineering, Iwate University, 4-3-5 Ueda, Morioka, Iwate 020-8551, Japan. E-mail: isao_taguchi@pref.aomori.lg.jp

*** Department of Mechanical Engineering Iwate University, 4-3-5 Ueda, Morioka, Iwate 020-8551, Japan
}

from 4.5 to 5.0 diameters from the wall. For the safe operation of nuclear reactors, Thadani and Peebles ${ }^{(4)}$ investigated the distribution of local void fraction in a bed of randomly packed uniform fuel spheres constructed in a typical model of pebble bed-type reactors.

All the above studies were experimental ones and focused on cylindrical containers. By simulating the packing of spherical particles into a cubic box, Furukawa et al. ${ }^{(5)}$ reported that the effect is up to 3.0 particle diameters from the wall or floor. It seems that this value resulted from combined effects from all the four walls and the floor, and that their virtual box was too small to obtain the accurate depth of influence.

Examining the statistical characteristics of a random packing of spheres produced by the same method as in Ref. (5), Kato et al. ${ }^{(6)}$ found that the packing structure is considerably affected by gravity; more precisely, line segments connecting the centers of spheres in contact lie more frequently around the direction $45^{\circ}$ from the vertical line, although they are uniformly distributed around the vertical line. Therefore, the ways the random packing structures are affected by the presence of the vertical wall and the presence of the horizontal floor must be different due to gravity.

In the present paper, we examine each distinct effect of the plane (not cylindrical) wall and floor on the random packing structures of a cubic aggregate of equal-sized spherical particles, for a wide range of cube sizes for bet- 


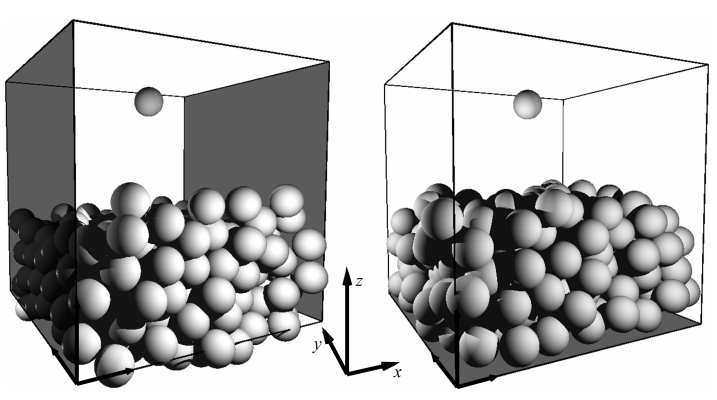

Fig. 1 Illustration of simulation conditions: Simulation (A) for wall effect on left side (shadowed walls affect structure) and Simulation (B) for floor effect on right (the shadowed floor affects structure)

ter accuracy of the simulation. We carry out the following two different random packing simulations.

- Simulation (A): We apply cyclic boundary conditions only in the $y$-direction along with random ups and downs of the floor to estimate the wall effect.

- Simulation (B): We employ cyclic boundary conditions in both of the horizontal directions to detect the floor effect.

By Simulation (A), we can find the effect of two opposite walls facing each other on packing, while by Simulation (B), we can find the effect of the floor on packing. Virtual cubic boxes for Simulations (A) and (B) are shown in Fig. 1. In this figure, the walls and floor are drawn in shadow. Using the obtained packing data from the simulations, we evaluate the wall or floor effect in terms of "area porosities" and the cumulative frequencies of the diameters of circles appearing on some cross sections created by cutting the aggregate at different levels, both as functions of distance from the wall or floor; the area porosity is defined as the ratio of the cross-sectional area occupied by vacancies to the whole aggregate cross-sectional area.

Furthermore, we employ two different ways of determining the area porosities, by using a very large aggregate and by averaging them over a large number of small ones. Comparing the results obtained from both methods, we clarify that the area porosities and their spatial variation are in good agreement with each other.

\section{Nomenclature}

$B$ : Box size, i.e., side length of virtual cubic box

$c$ : Viscosity constant

$D$ : Diameter of spherical particle

$\boldsymbol{d}$ : Overlapping depth vector between two spheres in collision

$g$ : Gravitational acceleration

$\boldsymbol{k}$ : Unit base vector along $z$-axis

$k$ : Spring constant between two or more particles in collision or against wall

$m$ : Mass of each particle

$\boldsymbol{x}$ : Position vector of falling sphere's center $\varepsilon:$ Small parameter to define settlement of each particle

$\phi$ : Volume (or bulk) porosity

$\phi_{A}$ : Area porosity

\section{Random Packing Simulation}

\subsection{Simulation method of random packing}

Various methods of constructing a computer simulation model for random packing are available (e.g., Refs. (5) - (8)). Among these methods, we use the method of rigid-sphere successive free fall in a container adopted in Ref.(6), because the method is one of the simplest ones and, in addition, it is appropriate for the construction of adequate microgeometry of the particle aggregate. The method assumes no bouncing, no bumping of precariously stable positions, and no consolidation by spreading spheres apart to accommodate incoming spheres; it neglects, furthermore, the acceleration terms in the equations of motion for each sphere. These assumptions make computing time short. The container into which spheres are dropped is cubic.

In simulation, with the $x$ and $y$ coordinates of the initial position of each sphere generated by pseudorandom numbers, the sphere is successively introduced only after the previous sphere has settled down in place. To see the wall effect in Simulation (A), we introduce the conventional cyclic boundary conditions only in the $y$-direction and displace the box floor up and down for each incoming sphere by magnitudes given by pseudorandom numbers to avoid possible packing regularity on the floor. For Simulation (B), we estimate the structural influence of the floor of the box in which spheres are accumulated by applying the cyclic boundary conditions both in the $x$ and $y$ directions and by keeping the floor at a constant level: see Fig. 1.

To simplify the algorithm, the settled spheres, which never move even if they are hit by a new sphere, are assumed to be elastic only when they collide with a falling sphere; the walls whose effects are considered are also assumed elastic with the same elastic constant for (A); the floor whose presence is taken into account is assumed rigid and remains unmoved for (B). No friction between the spheres and the walls and no particulate rotation are taken into consideration to save computing time. Therefore, each sphere rests at the position where gravity force is in equilibrium with the spring forces from the settled spheres (and the wall).

Because of the above simplification, the present simulation is applicable only in the case of spherical particles of metal or ceramics with a diameter of more than approximately $100 \mu \mathrm{m}$.

Consider a virtual box of $B \times B \times B$. In the box, each sphere with the diameter $D$ falls from the minimum height, which is larger than that of the settled sphere aggregate. The motion equation is given in vector form by 


$$
c \frac{d \boldsymbol{x}}{d t}=k \Delta \boldsymbol{d}-m g \boldsymbol{k},
$$

where $\boldsymbol{x}$ is the position vector of a falling sphere center, $\boldsymbol{k}$ is the unit vector along the $z$-axis which points upward and $\Delta \boldsymbol{d}$ is the overlapping depth vector of the sphere in collision with another still one. The notations $c, k, m$ and $g$ are the viscosity constant, the spring constant between two or more particles in collision, the mass of each particle and the gravitational acceleration, respectively. Adopting the spherical particle diameter $D$ as reference length, we introduce nondimensional variables and constant parameters: $\boldsymbol{X}=\boldsymbol{x} / D, \Delta \boldsymbol{D}=\Delta \boldsymbol{d} / D, T=m g t /(c D), K=D k /(m g)$. Thus, Eq. (1) can be rewritten in terms of these as follows.

$$
\frac{d \boldsymbol{X}}{d T}=K \Delta \boldsymbol{D}-\boldsymbol{k}
$$

To trace the fall of each sphere, we solve the equation of motion (Eq. (2)) by the Runge-Kutta-Gill method, which incorporates only gravity and spring forces. We judge that the sphere has stopped when its displacement during a given time increment $\Delta T$ is smaller than some given small value $\varepsilon$. We do not stop introducing spheres until the cubic region $0 \leq x \leq B, 0 \leq y \leq B, 0 \leq z \leq B$ is completely filled with particles. The pseudorandom numbers for the initial position of each sphere and for ups and downs of the box floor level are generated by Wichmann and Hill's $\operatorname{method}^{(9)}$.

In running our program, we have used the following numerical values: time increment $\Delta T=1.0 \times 10^{-3}$, spring constant $K=1.0 \times 10^{3}$ and small parameter $\varepsilon=1.0 \times 10^{-7}$, as employed by Kato et al. ${ }^{(6)}$

\subsection{Reality of result of present method}

In Ref.(6), using the packing data obtained by the same simulation method, it was found that the constructed random packings are transversely isotropic in terms of packing structure and that Young's modulus in the vertical direction is larger than that in the horizontal directions by approximately 17\%. Furthermore, experimentally, the phase velocities of the first compressional and shear waves through the sintered glass-bead plates saturated with water are considerably larger in the direction of gravity acting in sintering than in the horizontal direction. Thus, it was shown that the sintered aggregates are transversely isotropic in both the simulation and the experiment. Therefore, this simulation method reflects reality quantitatively well in spite of its simplicity.

\subsection{Case of no wall/floor effect}

As test runs, we have carried out preliminary random packing simulations by applying both the cyclic boundary conditions both in the $x$ and $y$ directions and the floor ups and downs; this is the case of no wall/floor effect; that is, the case of an infinite cubic box.

For all four box sizes $B / D=20,60,100$ and 140 prepared, we have made three runs using three different sets of pseudorandom numbers. For all three aggregate sam-
Table 1 Porosities $\phi$ and area porosities $\phi_{A}$ of 3 samples for each of four box sizes; their means and standard deviations, and mean number of spheres contained in each

\begin{tabular}{|c|c|c|c|c|c|}
\hline \multirow{2}{*}{\multicolumn{2}{|c|}{$\begin{array}{c}\text { Box size } \\
\text { Mean number of spheres }\end{array}$}} & $B / D=20$ & $B / D=60$ & $B / D=100$ & $B / D=140$ \\
\hline & & 9343 & 244452 & 1124386 & 3076826 \\
\hline \multirow{5}{*}{$\phi$} & Sample 1 & 0.418551 & 0.417006 & 0.417054 & 0.417086 \\
\hline & Sample 2 & 0.416994 & 0.417110 & 0.417122 & 0.417016 \\
\hline & Sample 3 & 0.416933 & 0.417123 & 0.417067 & 0.417050 \\
\hline & Mean & 0.417493 & 0.417079 & 0.417081 & 0.417051 \\
\hline & Standard deviation & 0.000916 & 0.000063 & 0.000036 & 0.000034 \\
\hline \multirow{5}{*}{$\phi_{A}$} & Sample 1 & 0.418133 & 0.416659 & 0.417442 & 0.417128 \\
\hline & Sample 2 & 0.418377 & 0.417645 & 0.416943 & 0.417042 \\
\hline & Sample 3 & 0.417333 & 0.417403 & 0.417243 & 0.417029 \\
\hline & Mean & 0.417948 & 0.417236 & 0.417209 & 0.417066 \\
\hline & Standard deviation & 0.000546 & 0.000514 & 0.000251 & 0.000053 \\
\hline
\end{tabular}
of 3 samples

ples for each box size, bulk porosities $\phi$, their mean and standard deviation, and area porosity $\phi_{A}$ averaged over all cross sections at the distance of all integral multiples of $D$ from the virtual floor have been calculated from lists of the three coordinates of each sphere's center; these are shown in Table 1.

The average porosity of the random packings constructed by the present runs is approximately $\phi=0.417$, independent of box size if we take three digits as important figures. This mean value is in good agreement with that obtained by Kato et al. ${ }^{(6)}$

In general, random packings are separated into three categories ${ }^{(10)}$. "Very loose random packing" is obtained when momentum is minimized and opportunities for bridging are maximized. For example, when the fluid velocity in a fluidized bed is slowly reduced, spheres settle to a mean porosity of approximately $\phi=0.44$. "Loose random packing" $(0.41 \leq \phi \leq 0.44)$ is obtained by dumping and in some cases by tapping the container, which gives the spheres more momentum but provides little opportunity for bridging. "Close random packing" $(\phi \approx$ 0.36 ) is produced after the container is well shaken, i.e., when there is considerable momentum and little chance of bridging. The porosity $\phi=0.417$ obtained here is rather small in the range of loose random packing, because the present method allows no bridging.

Furthermore, the averaged area porosity is also approximately $\phi_{A}=0.417$. It is theoretically known that the area porosity $\phi_{A}$ is identical to the bulk porosity $\phi$ for a random packing aggregate of "infinite extent." As expected, the larger the box is, the smaller the standard deviation is. This implies that the box into which spheres are deposited needs to be larger than a certain size even if both the cyclic boundary conditions and the random ups and downs of the floor level for each incoming sphere are employed.

The aggregate of box size $B / D=20$ is composed of approximately 9000 spheres, while the largest box of size 
$B / D=140$ contains approximately three million spheres; the computing time we spent and the memory we used ensure that even a common PC (Pentium 4) can perform the simulation under consideration without difficulty.

\section{Effect of Wall and Floor on Packing Structures}

Following the above-mentioned procedures, we have carried out two different random packing simulations by applying (A) cyclic boundary conditions only in the $y$ direction and random ups and downs of the floor to estimate the effect of an $x=0$ wall and (B) cyclic boundary conditions in both of the horizontal directions to see the effect of a $z=0$ floor. As a result, we have obtained a list of the coordinates of each settled spherical particle's center for a wide range of virtual box sizes. From each list, we have extracted the statistical values suitable for estimating the wall/floor effects: area porosity and cumulative frequency of diameters of the circles on some cross section made by cutting the aggregate at some different level.

\subsection{Area porosity}

To examine how deeply the wall/floor effect penetrates the aggregate and how the presence of the wall/floor regularizes the random packing structure near it, the area porosity $\phi_{A}$ as a function of the distance of associated cross sections from the wall/floor has been obtained from the packing data; the area porosity $\phi_{A}$ is defined as the ratio of the cross-sectional area occupied by vacancies to the whole cross-sectional area.

Fluctuations of the area porosities with increasing distance from the wall/floor are calculated from the lists of sphere center positions obtained from Simulations (A) and (B) as functions of the $x$ or $z$ coordinate of the respective $x=$ constant or $z=$ constant cross section on which the area porosities are measured. The area porosities versus distance from the wall/floor are shown in Figs. 2 and 3, corresponding to (A) and (B), for the box size $B / D=20$. We can see from these figures that the area porosity at the wall $x / D=0$ or on the floor $z / D=0$ starts with the value of unity as expected and falls down to a minimum value of approximately 0.34 at a distance of approximately a half particle diameter from the surface; then, it rises up to the first and only "sharp" peak at exactly $z / D=1$. Moving away from the peak, the area porosity irregularly oscillates around its mean value. We can also see from Fig. 2 that, as the opposite wall is approached, the area porosity varies in exactly the same way as for the left wall.

The left sides of Figs. 2 and 3 are magnified and shown in Figs. 4 and 5, respectively. In these figures, the curves for $B / D=60,100$ and 140 are also added to the curve of $B / D=20$. The area porosities are almost the same for all box sizes near the wall or floor, implying the tendency that the presence of the wall/floor makes the packing more regular. The area porosity fluctuations decrease with increasing box size in the deeper region where the

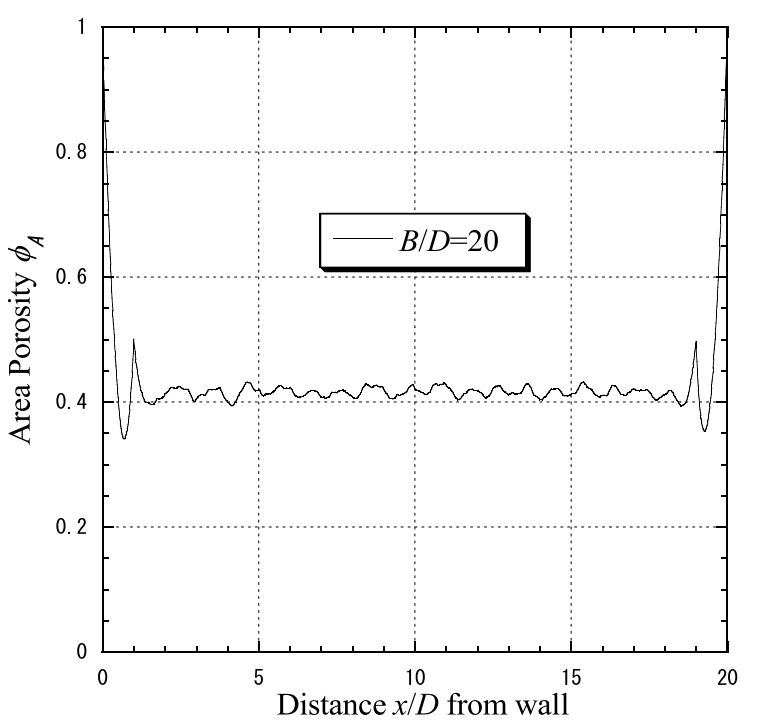

Fig. 2 Fluctuation of area porosity along $x$ coordinate of cross section on which it is calculated (Simulation (A) for wall effect)

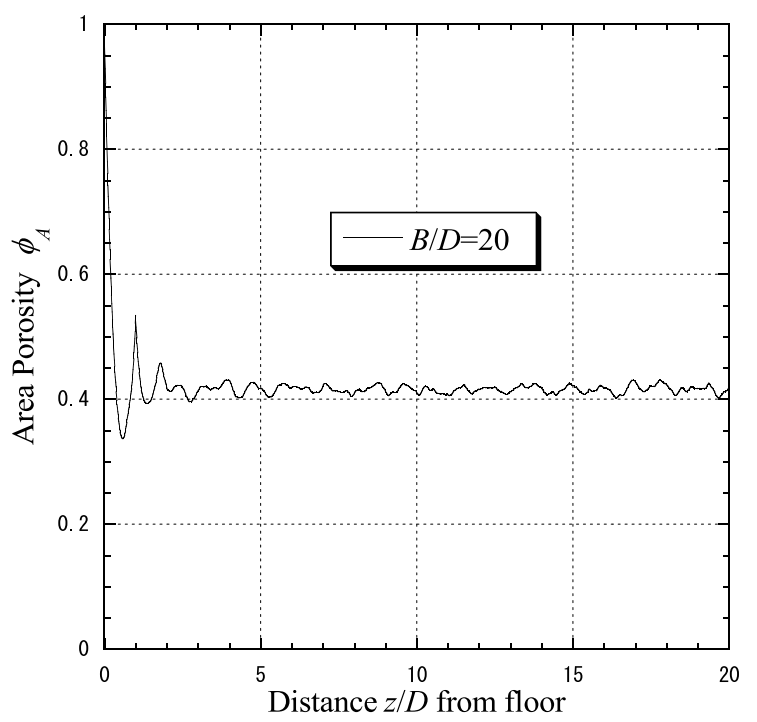

Fig. 3 Fluctuations of area porosity along $z$ coordinate of cross section on which it is calculated (Simulation (B) for floor effect)

randomness of packing prevails. Comparison of these two figures for the largest box $(B / D=140$, depicted by solid curves) reveals that the area porosity fluctuations in Fig. 5 are larger in amplitude and penetrate deeper than those in Fig. 4. That is, the influence from the floor dominates over that from the wall, as will be discussed later from another point of view.

It is interesting to compare the simulated curves in Fig. 4 with the experimental ones in Refs.(1)-(4), although the latter used a cylindrical container filled with spherical particles instead of a cubic one; for these experimental results, the area porosities should be read as the average porosities over a very thin cylindrical shell volume 


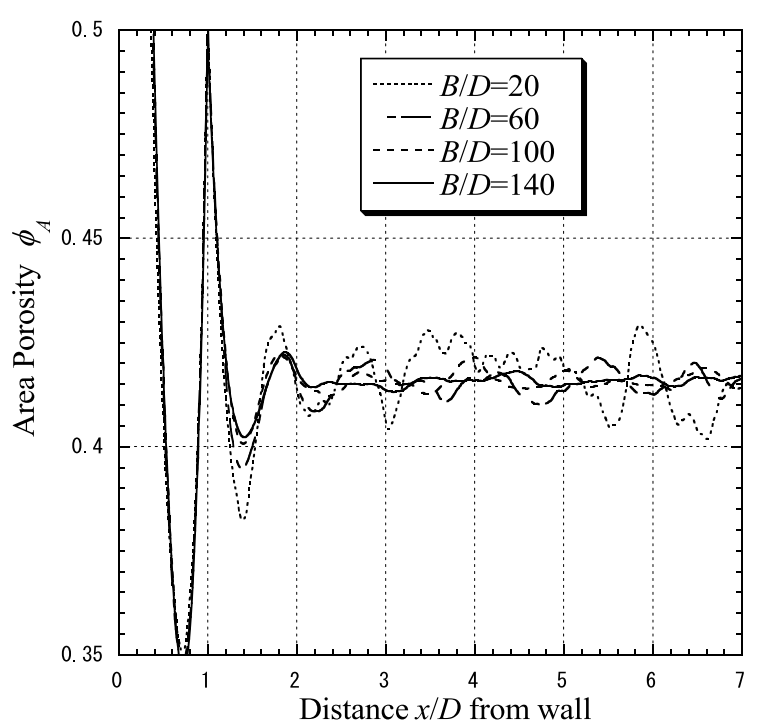

Fig. 4 Magnified fluctuations of area porosity along $x$ coordinate of cross section on which it is calculated, for four box sizes (Simulation (A) for wall effect)

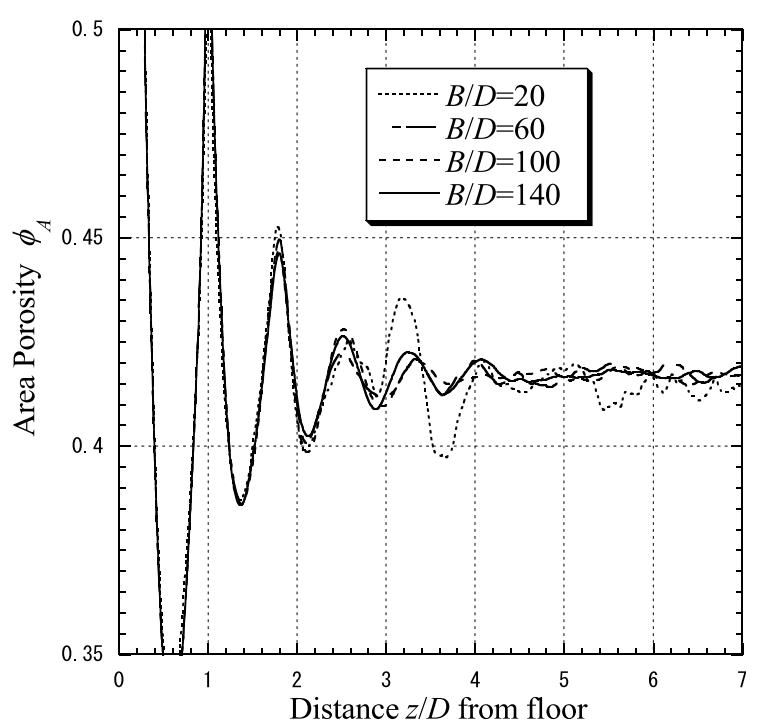

Fig. 5 Magnified fluctuations of area porosity along $z$ coordinate of cross section on which it is calculated, for four box sizes (Simulation (B) for floor effect)

cut from a cylindrical packing aggregate. The damped oscillatory behavior starting from unity is common to the experimental results and the present ones. However, the experimental curves of porosity have alternate maxima and minima around several integral multiples of the particle radius from the cylindrical wall ${ }^{(4)}$; the present simulations show that there are only a few or a little more of the maxima and minima for the case of wall effects (Fig. 4), while about a dozen maxima and minima appear for the case of the floor effects in Fig. 5 (Here again, we can say that the influence of the floor dominates over that of the wall). Furthermore, all the above experiments show no sharp peak at $x / D=1$ because of the values averaged over a small shell thickness as mentioned above; in contrast, both of these figures have a sharp peak at $x / D$ or $z / D=1$.

It can be also seen from Figs. 4 and 5 that every peak of the alternate maxima and minima for $z / D>1.0$ shifts from the point in integral multiples of particle radius $D / 2$ toward the wall/floor, although the first peak of the maxima lies exactly on $z / D=1$. The magnitude of the shift increases with the distance of their peaks from the wall/floor. This can be understood from the fact that the first layer of spherical particles lies exactly on the wall/floor so that it is a perfect layer with no irregularity in the normal direction, while protrusions of the second or further layers are caught in the indentations of the first or previous layer, as the peaks shift toward the wall/floor. Furthermore, this disturbs the arrangement of spheres more and decreases peak height with increasing distance from the wall/floor. This type of shift can be seen in Refs. (2) and (3), which contradicts the figure in Ref. (4).

The extent of the wall effect was experimentally concluded to be up to 3.0 particle diameters from the wall in Ref. (4) and 4.5 to 5.0 diameters in Ref. (3). The previous simulation ${ }^{(5)}$ showed that the effect is up to 3.0 particle diameters from the wall or floor. It seems that this value stemmed from the combined effects from all four walls and the floor. The present results shown in Figs. 4 and 5 suggest approximately 2.5 particle diameters from the wall and approximately 4.5 particle diameters from the floor as depths of influence. These values come from the observations that the curves for $B / D=140$ are almost flat for $x / D$ or $z / D$ beyond these values, as judged by observation. It should be also emphasized that the effects of the wall and the floor are different, which is certainly due to gravity.

\subsection{Cumulative frequency of diameters of circles appearing on cross sections}

For the same purpose as that described in the previous subsection, we have calculated the cumulative frequencies of the diameters of circles appearing on some cross sections created by cutting the aggregate at different levels. If a randomly packed aggregate of spheres is sectioned along a plane, a large number of circles will appear on its resultant cross section; the circles have various values of diameter $d$, which is naturally smaller than or equal to $D$. The cumulative frequency of the diameter $f(d / D)$ is theoretically given by

$$
f(d / D)=1-\sqrt{1-(d / D)^{2}},
$$

provided that the centers of packed spheres are distributed in a uniformly random manner ${ }^{(8),(11)}$.

The simulated cumulative frequency of nondimensionalized diameters $d / D$ for various cross sections near the wall and floor are shown in Figs. 6 and 7, respectively, together with the theoretical prediction drawn by solid curves and given by Eq. (3). Naturally, the frequency 


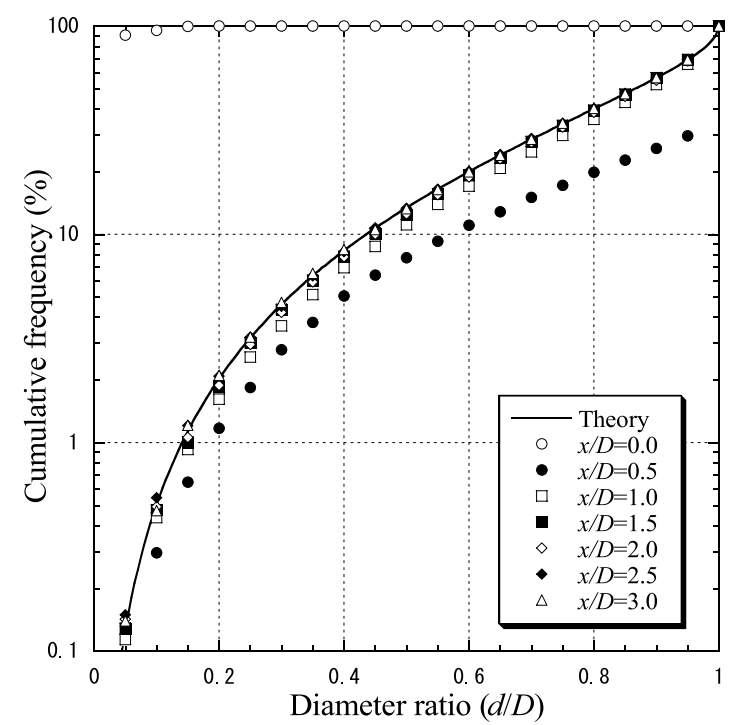

Fig. 6 Cumulative frequencies of diameters of circles appearing on various $x=$ constant cross sections near wall together with theoretical prediction $(B / D=140)$

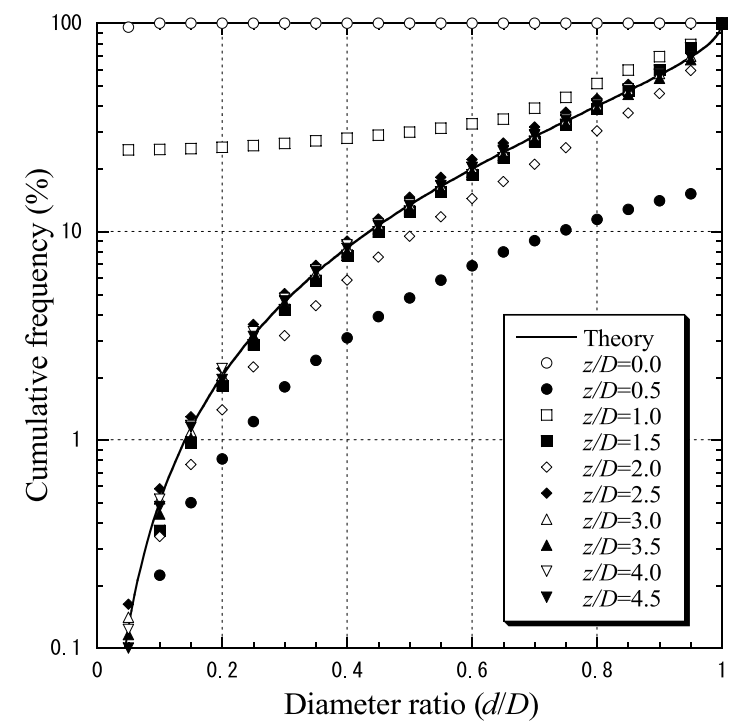

Fig. 7 Cumulative frequencies of diameters of circles appearing on various $z=$ constant cross sections near wall together with theoretical prediction $(B / D=140)$

monotonically increases with $d / D$ for any of the positions taken by the aggregate cross section (except just on the wall or floor) and reaches $100 \%$ at $d / D=1$. The cumulative frequency just on the wall or floor $(x / D=0$ or $z / D=0$ ), which is depicted by hollow circles in the figures, is $100 \%$ for all diameter ratios because all diameters vanish there.

The curve for the cumulative frequency at the depth of one particle diameter $(x / D=1.0)$ in Fig. 6 is significantly different from its counterpart $(z / D=1.0)$ in Fig. 7 ; both are depicted by small hollow squares. This difference can be understood as follows. In the latter floor effect case, at an early stage of sphere packing, each sphere falls onto the

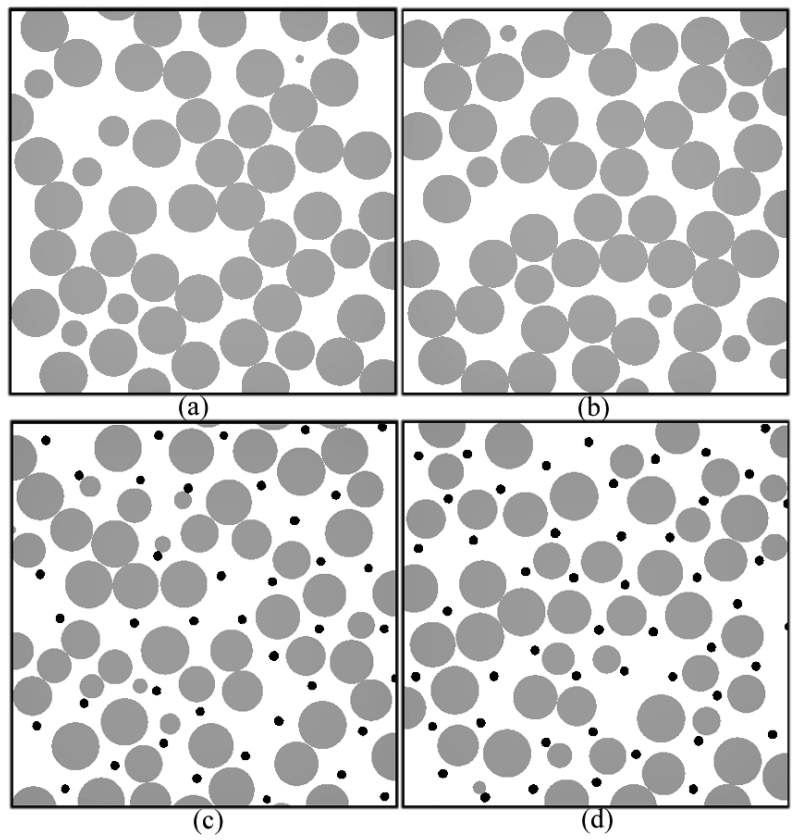

Fig. 8 Illustration of cross sections of $x / D$ or $z / D=0.5$ shown in (a) or (b), respectively, and $x / D$ or $z / D=1.0$ in (c) or $(\mathrm{d}),(B / D=8)$; spheres' cross sections are shadowed and infinitesimal ones are magnified to pitch-black dots

flat floor $z / D=0$ from its initial position assigned by a pair of uniform random numbers so that all spheres settled on the floor are uniformly and densely distributed over it. In contrast, in the former wall effect case, each sphere falls onto already randomly settled spheres even just near the wall; some of the settled spheres are in contact with the wall and some are located just near the wall. Thus, more spheres are in contact with the floor than with the vertical wall.

Let us see the difference between these two cases by illustrating two cross sections of a box of $B / D=8$ for (A) and (B) in Fig. 8; the grey circles show the circular cross sections of spheres on the cross sections of $x / D=0.5$ and 1.0 ((a) and (c): wall effect) and $z / D=0.5$ and 1.0 ((b) and (d): floor effect). We can see from this figure that the circles with a large diameter or with a diameter of almost $d / D=1.0$ are predominant in number on the cross section of $x / D=0.5$ or $z / D=0.5$ (on the top). This is because the spheres in contact with the wall or floor are sectioned into halves through their centers. Thus, on this section, since the smaller circles are much fewer than those of approximately $d / D=1.0$, the cumulative frequencies depicted by the solid circles in Figs. 6 and 7 remain below the theoretical curve for the whole range of $d / D<1$.

The illustrations on the bottom in Fig. 8 show the section of $x / D=1.0$ or $z / D=1.0$. Here, we can see a number of small pitch-black dots, almost all of which depict the tops of spheres in contact with the wall/floor surface (Since the tops" "sections" of spheres have infinitesimal, 
thus invisible, diameters, we magnify them up to the size of dots). To see the contiguity of the spheres to the surface, the number of spheres in contact with the floor or wall (or with their centers existing at $x / D=0.5$ or $z / D=0.5$ ) has been counted. In Fig. 8 for the case of $B / D=8$, the number of spheres in contact is 49 with the floor, whereas 39 with the wall. For the case of $B / D=20$, the number of spheres in contact is 310 with the floor on average, whereas 220 with the wall. This number of 310 for the floor is considered rather large, if compared with 400 for the regular square arrangement of spheres on a $20 \times 20$ floor. This supports the above argument about the difference in cumulative frequency for $x / D=0.5$ or $z / D=0.5$ and also shows that the spheres are more densely distributed on the floor compared with those on the wall.

From Figs. 6 and 7, it can be seen that all frequency curves for $x / D=1.5,2.0,2.5$ and 3.0 and for $z / D=1.5$ to 4.5 except $z / D=2.0$ are very close to the theoretically predicted curve and these seem closer for the larger $x / D$ and $z / D$. This is the true, although the range of these $x / D$ 's and $z / D$ 's is still in the zone affected by the wall or floor. This closeness may be associated with the abovementioned shift of peaks (maxima and minima) toward the surface. The further the cross section is from the wall or floor the closer the curve is to the theoretical prediction, because the randomness of packing is more dominant; that is, the wall/floor effect vanishes there.

\subsection{Average of area porosities over a number of samples}

If there is no wall/floor effect, the fluctuations in area porosity against the position of the cross section of finite extent on which the area porosity is estimated must be completely random. It is clear that the area porosity averaged over increasing number of samples approaches the "true" area porosity, i.e., the porosity in an infinite sample. In contrast, the presence of the wall or floor might regularize the packing structure. Therefore, the area porosity near the wall/floor does not reach the true area porosity even for a very large sample.

In addition to the cases of $B / D=20,60,100$ and 140 , we have calculated the area porosity averaged over 100 samples for $B / D=14$. Here, we have selected $B / D=14$ for the "effective area" of 100 cross sections to be equal to the area for $B / D=140$.

Figures 9 and 10 show the fluctuations of this averaged area porosity and the porosities of a single sample for $B / D=20$ and 140 .

From these figures, we can see that the whole damped amplitude behavior of the porosity averaged over many small samples is in good agreement with that of the nonaveraged porosity for a single, very large sample, which seems similar to ergodicity. In contrast, these two curves markedly differ from the one for the single $B / D=20$ sample; we cannot extract the wall/floor effect from such a

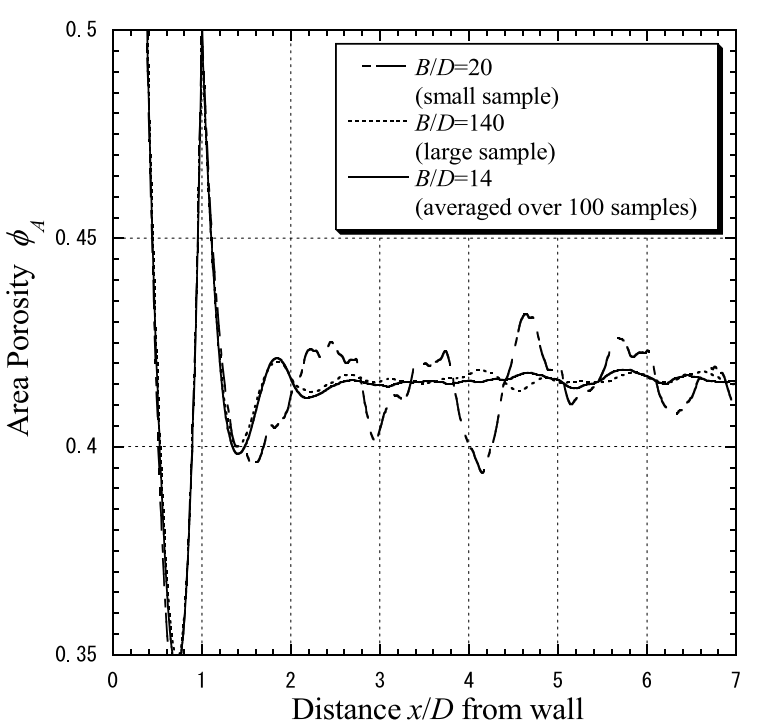

Fig. 9 Comparison between area porosity fluctuations for single large and small sample and those averaged over a number of small samples (Simulation (A) for wall effect)

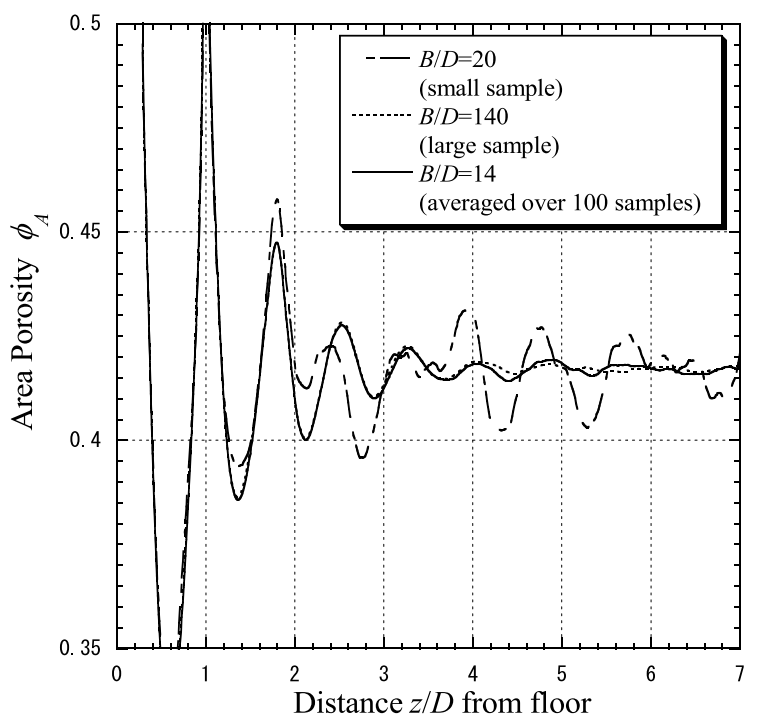

Fig. 10 Comparison between area porosity fluctuations for single large and small sample and those averaged over a number of small samples (Simulation (B) the floor effect)

single small sample.

As shown in subsection 3.3, a box of large size is necessary for simulation with a high accuracy even if both the cyclic boundary conditions and the random ups and downs of the floor level for each incoming sphere are employed. It is not easy to carry out the simulation to construct and examine such a large aggregate sample using a personal computer, leading to long computing time and very large memory. Thus, to avoid this difficulty, we should average the variation of area porosity over a large number of rather small samples; this is allowed because the simulated area porosities have properties similar to ergodicity.

It is also found in Figs. 9 and 10 that the wall/floor 
effect reaches as deep as 2.5 particle diameters from the wall and about 4.5 diameters from the floor.

\section{Conclusions}

By random packing simulation of equal-sized spherical particles, we examined the effect of the wall and floor of a container on packing structure, which is shown as a function of distance from the wall or floor in terms of the area porosity and the cumulative frequency of diameters of the circles appearing on some cross section. Although the extent of the wall effect was experimentally concluded to be up to 3.0 or 4.5 to 5.0 particle diameters from the wall, our conclusions are as follows.

(1) The depth of the wall effect is up to 2.5 particle diameters from the wall, while that of the floor effect is up to 4.5 particle diameters from the floor.

(2) There is a considerable difference in packing structure between the area near the wall and that near the floor, due to gravity.

(3) The whole damped amplitude behavior of area porosity averaged over many small samples is in good agreement with that of the nonaveraged porosity for a single, very large sample; this means that the packing structure reflecting the wall and floor effect has properties similar to ergodicity.

(4) There is a significant difference in packing structure between areas near and far from the wall/floor: regularity and randomness.

\section{References}

( 1 ) Roblee, L.H.S., Baird, R.M. and Tierney, J.W., Radial Porosity Variations in Packed Beds, A. I. Ch. E. J., Vo.4 (1958), pp.460-464.
( 2 ) Macrae, J.C. and Gray, W.A., Significance of the Properties of Materials in the Packing of Real Spherical Particles, British Journal of Applied Physics, Vol.12 (1961), pp.164-172.

( 3 ) Benenati, R.F. and Brosilow, C.B., Void Fraction Distribution in Beds of Spheres, A. I. Ch. E. J., Vol.4 (1962), pp.359-361.

(4) Thadani, M.C. and Peebles, F.N., Variation of Local Void Fraction in Randomly Packed Beds of Equal Spheres, I\&CE Process Design and Development, Vol.5 (1966), pp.265-268.

( 5 ) Furukawa, K., Imai, K. and Kurashige, M., Simulated Effect of Box Size and Wall on Porosity of Random Packing of Spherical Particles, Acta Mechanica, Vol.140 (2000), pp.219-231.

( 6 ) Kato, H., Matsunaga, C., Kurashige, M. and Imai, K., Anisotropy in Packing Structure and Elasticity of Sintering Spherical Particles, JSME Int. J., Ser. A, Vol.45, No.4 (2002), pp.585-595.

( 7 ) Yen, K.Z.Y. and Chaki, T.K., A Dynamic Simulation of Particle Rearrangement in Powder Packings with Realistic Interactions, Journal of Applied Physics, Vol.71 (1992), pp.3164-3173.

( 8 ) Bennett, C.H., Serially Deposited Amorphous Aggregates of Hard Spheres, Journal of Applied Physics, Vol.43 (1972), pp.2727-2734.

(9) Wichmann, B.A. and Hill, I.D., An Efficient and Portable Pseudo-Random Number Generator, Royal Statistical Society, Algorithm as 183, (1982), pp.188190.

(10) Haughey, D.P. and Beveridge, G.S.G., Structural Properties of Packed Beds, Can. J. Chem. Eng., Vol.47 (1969), pp.130-140.

(11) Debbas, D. and Rumpf, H., On the Randomness of Beds Packed with Spheres or Irregular Shaped Particles, Chem. Engrg. Sci., Vol.21 (1966), pp.583-607. 